\title{
Dependence of quantum-Hall conductance on the edge-state equilibration position in a bipolar graphene sheet
}

\author{
Dong-Keun $\mathrm{Ki}^{1}$, Seung-Geol Nam ${ }^{1}$, Hu-Jong Lee ${ }^{1,2}$ * and Barbaros Özyilmaz ${ }^{3}$ \\ 1 Department of Physics, Pohang University of Science and Technology, Pohang 790-784, Republic of Korea \\ ${ }^{2}$ National Center for Nanomaterials Technology, Pohang 790-784, Republic of Korea \\ ${ }^{3}$ Department of Physics, National University of Singapore, Singapore 117542
}

(Dated: November 10, 2018)

\begin{abstract}
By using four-terminal configurations, we investigated the dependence of longitudinal and diagonal resistances of a graphene $\mathrm{p}-\mathrm{n}$ interface on the quantum-Hall edge-state equilibration position. The resistance of a p-n device in our four-terminal scheme is asymmetric with respect to the zero point where the filling factor $(\nu)$ of the entire graphene vanishes. This resistance asymmetry is caused by the chiral-direction-dependent change of the equilibration position and leads to a deeper insight into the equilibration process of the quantum-Hall edge states in a bipolar graphene system.
\end{abstract}

PACS numbers: 73.43.Fj, 71.70.Di, 73.61.Wp, 73.23.-b

Half-integer quantum-Hall (QH) effect in graphene manifests the massless-Dirac-fermionic nature of carriers in the material. $\stackrel{1.2}{=}$ The corresponding QH edge state in graphene has been explicitly confirmed to be chiral ${ }^{3}$ as in an ordinary two-dimensional (2D) electron gas. $\underline{.4}^{4,5}, \underline{6}$ Since carriers in graphene possess bipolar characteristics, the filling factor $(\nu)$ and the chiral direction of the edge states can be modulated by gating a graphene sheet electrostatically $\stackrel{1,2}{\Perp}$ Although the spatial deflection of an edge state is hard to be realized, $\frac{7}{,}$ a partial reflection of the edge state can be accomplished in locally gated graphene devices by means of the edge-state equilibration at p-n interfaces $\frac{8.9,10,11}{}$ Nonetheless, previous conductance measurements, either in two-terminal ${ }^{8,9,10}$ or in four-terminal configurations, $\stackrel{11}{=}$ did not explicitly reveal the dependence of the conductance on the equilibration position (EP), where the edge states with different filling factors are equilibrated.

Recently, by measuring four-terminal magnetoresistance of a spatially chemical-doped graphene p-n device, the longitudinal resistances are shown to be either quantized at finite values or vanish depending on the gate voltage, the measurement configuration, and the magnetic field $(H)$ direction. ${ }^{12}$ Analysis of the data confirmed that the four-terminal magnetoresistance depends on the location of the EP. From this result, one may suppose that the location of the EP can be experimentally determined simply by measuring four-terminal longitudinal magnetoresistance in any hybrid systems. In the study, however, the $\nu$ of the chemically doped region was not flexibly controllable, as distinct from the locally top-gated devices, where the $\nu$ of the top-gated region can be controlled separately from the region of the global gating only. In this study, we took the longitudinal $\left(R_{L}\right)$ and the diagonal $\left(R_{D}\right)$ resistances of a graphene p-n interface established by a local top-gate in four-terminal configurations. The measurement scheme enabled us to access in detail the development of the equilibration condition of the $\mathrm{QH}$ edge conducting states in graphene. We locate the EP's corresponding to different measurement configurations and explicitly show that both four-terminal $R_{L}$ (a)

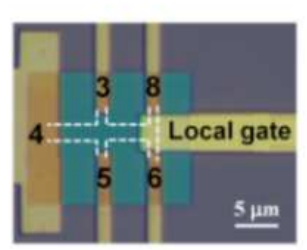

(c) Configuration 1

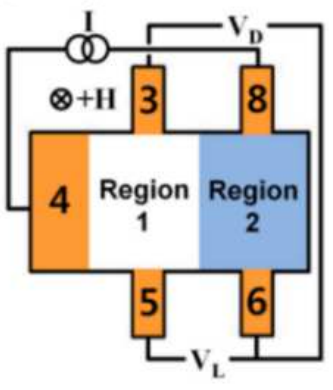

(b)

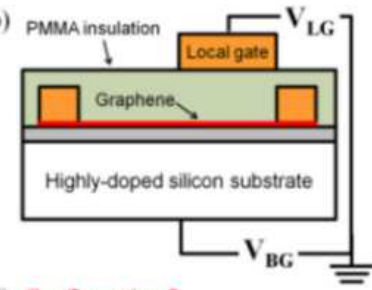

(d) Configuration 2

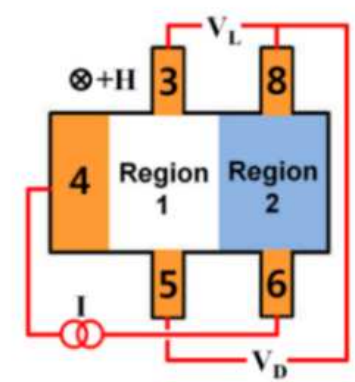

FIG. 1: (Color online) (a) Optical image of the sample. A PMMA insulation layer covers the graphene sheet, the boundary of which is defined by white broken lines. The white scale bar represents $5 \mu \mathrm{m}$. (b) Schematic gate configuration. (c) Measurement configuration 1. (d) Measurement configuration 2.

and $R_{D}$ are sensitive to the location of the EP.

A graphene p-n device was fabricated by electronbeam patterning and depositing a $\mathrm{Cr}(5 \mathrm{~nm}) / \mathrm{Au}(25$ $\mathrm{nm}$ ) bilayer on a mechanically exfoliated mono-layer graphene sheet $\underline{13}$ It was then followed by the polymethyl methacrylate (PMMA; $950 \mathrm{~K}, 2 \%$ in anisole) insulation 14 and the deposition of a local-gate, which covered one side of the sample $\mathrm{e}^{11}$ [Figs. 1(a,b)]. The device was cooled down to $120 \mathrm{mK}$ in a dilution fridge and $R_{D}\left(=V_{D} / I\right)$ and $R_{L}\left(=V_{L} / I\right)$ were obtained in two different measurement configurations [Figs. 1(c,d)] by using the conventional lock-in technique 11 The bipolar character of the device was verified at zero magnetic field $H$ by measuring $R_{L}$ as a function of local-gate and back-gate voltages 

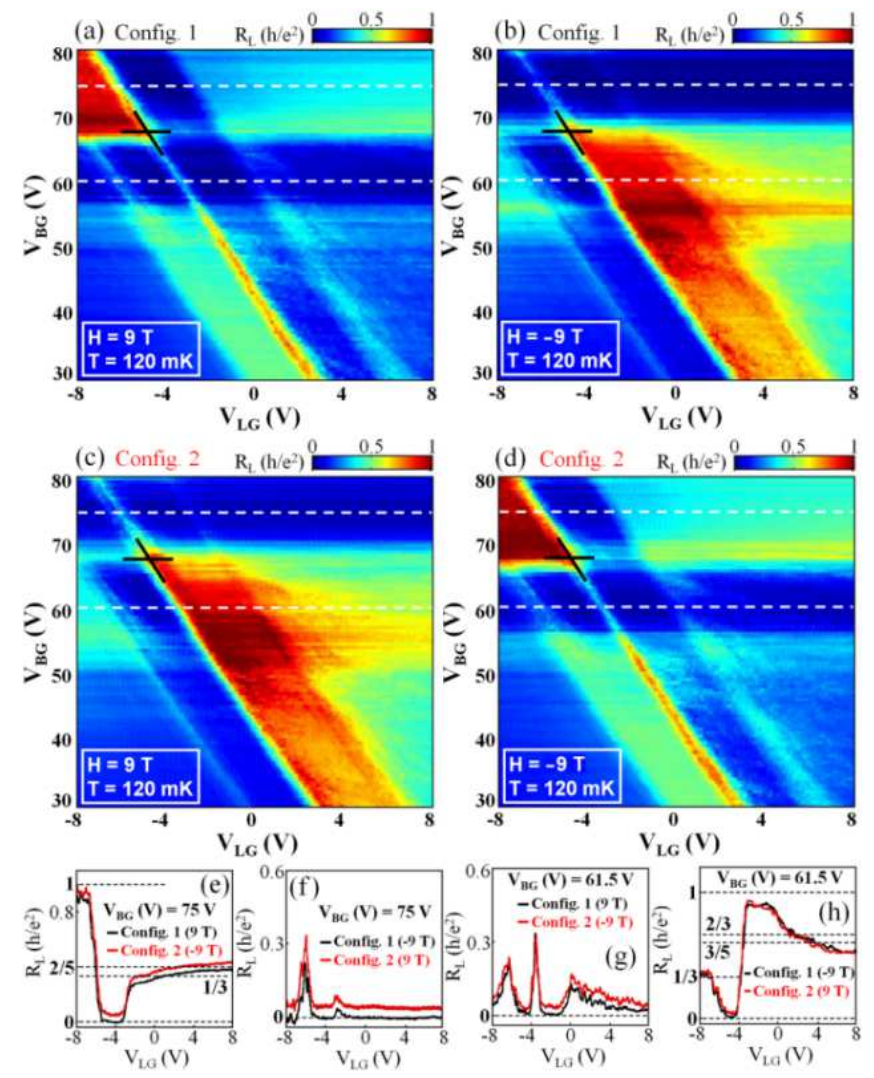

FIG. 2: (Color online) (a,b) 2D contrast maps of $R_{L}\left(V_{L G}, V_{B G}\right)$ for the configuration 1 , and $H=9 \mathrm{~T}$ and -9 $\mathrm{T}$, respectively. (c,d) $2 \mathrm{D}$ contrast maps of $R_{L}\left(V_{L G}, V_{B G}\right)$ for the configuration 2 , and $H=9 \mathrm{~T}$ and $-9 \mathrm{~T}$, respectively. White broken lines in (a-d) indicate the values of $V_{B G}$ where the slice data in (e-h) are extracted. (e,f) Slice plots of $2 \mathrm{D}$ contrast maps at $V_{B G}=75 \mathrm{~V}$. (g,h) Slice plots of $2 \mathrm{D}$ contrast maps at $V_{B G}=61.5 \mathrm{~V}$. Black and gray lines in (e-h) represent the slice data for the configurations 1 and 2 , respectively.

( $V_{L G}$ and $V_{B G}$, respectively), which exhibits a cross-like structure in the parameter space set up by the local variation of the density and the type of conduction carriers 11 (not shown).

The $2 \mathrm{D}$ contrast maps in Fig. 2 display $R_{L}\left(V_{L G}, V_{B G}\right)$ measured in two different configurations at $H= \pm 9 \mathrm{~T}$. Figs. 2(a-d) have several skewed blocks with varied contrast, i.e., with differently quantized resistances. Each figure has an asymmetry with respect to the zero point (a black cross), where the value of $\nu$ of the $\mathrm{QH}$ edge states in the regions $1\left(\nu_{1}\right)$ and $2\left(\nu_{2}\right)$ vanishes. The region 1 (2) represents the area outside (underneath) the local gate [Figs. 1(c,d)]. Figure 2(a), which corresponds to the configuration 1 and $H=+9 \mathrm{~T}$, is analogous to Fig. 2(d) for the configuration 2 and $H=-9$ T. Similarly, Fig. 2(b) nearly duplicates Fig. 2(c). On the other hand, resistances for opposite configurations at a given $H$ [Figs. 2(a,b) or Figs. 2(c,d)] have $180^{\circ}$-rotational symmetry with respect to the zero point. This is in contrast to earlier two-terminal studies, where conductances
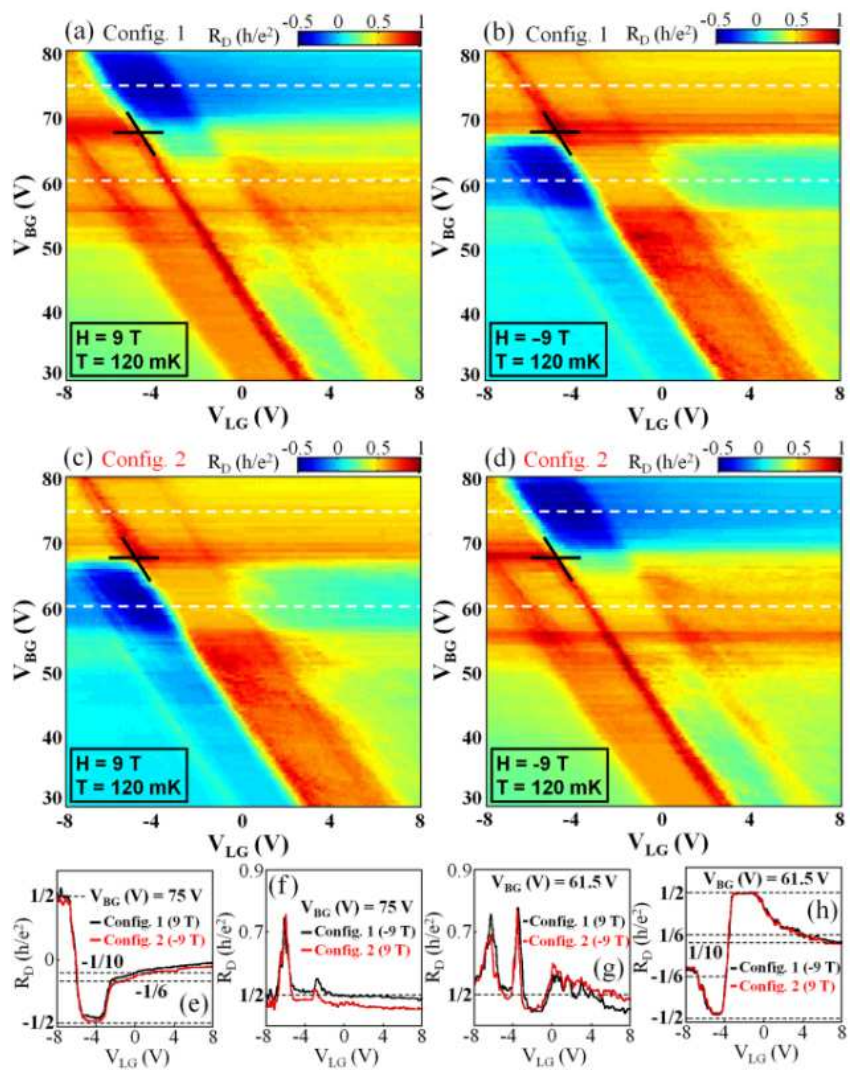

FIG. 3: (Color online) (a,b) 2D contrast maps of $R_{D}\left(V_{L G}, V_{B G}\right)$ for the configuration 1 , and $H=9 \mathrm{~T}$ and -9 $\mathrm{T}$, respectively. (c,d) $2 \mathrm{D}$ contrast maps of $R_{D}\left(V_{L G}, V_{B G}\right)$ for the configuration 2 , and $H=9 \mathrm{~T}$ and $-9 \mathrm{~T}$, respectively. White broken lines in (a-d) indicate the values of $V_{B G}$ where slice data in (e-h) are extracted. (e,f) Slice plots of $2 \mathrm{D}$ contrast maps at $V_{B G}=75 \mathrm{~V}$. (g,h) Slice plots of $2 \mathrm{D}$ contrast maps at $V_{B G}=61.5 \mathrm{~V}$. Black and gray lines in (e-h) represent the slice data for the configurations 1 and 2 , respectively.

were always symmetric, $\stackrel{8,10}{, 1}$ as well as to the four-terminal studies on a p-n-p device, where only $R_{D}$ exhibited an asymmetry: 11

Details of resistance variations can be analyzed by examining slices of Figs. 2(a-d) at two different values of $V_{B G}$, as shown in Figs. 2(e-h). Each figure contains two such slices black and gray lines for a concurrent change of the measurement configuration and the $H$ direction. As expected, black and gray lines in each slice are almost identical. $R_{L}$ 's in Figs. 2(f,g) oscillate with zero resistance plateaus, which are the Shubnikov-de Hass oscillations in the region 2. However, $R_{L}$ 's in Figs. 2(e,h) show a few plateaus, some with fractional resistances. This is an indication of the finite edge-state reflection at the p-n interface 11 One notes that the difference between Fig. 2(e) [Fig. 2(h)] and Fig. 2(f) [Fig. 2(g)], which are in an identical configuration, is in the $H$ direction only. It will be shown below that this seeming strange feature arises from the sensitivity of a four-terminal resistance on the location of the EP in our graphene p-n device. 


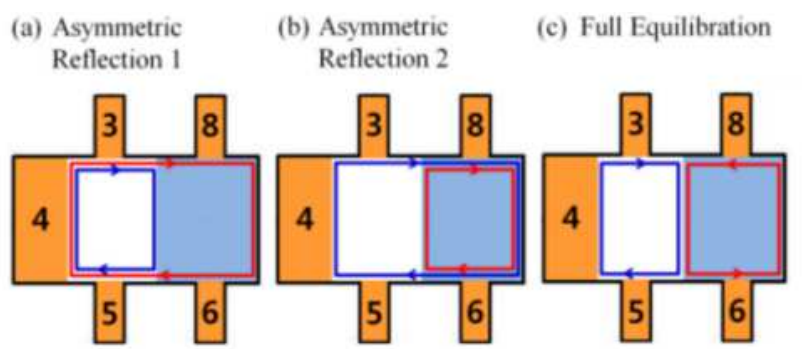

(d) Fully covered case

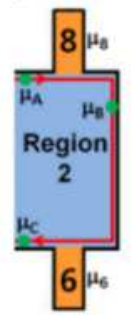

(e) Partially covered case

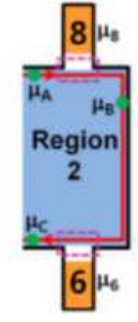

FIG. 4: (Color online) Schematic configurations of the edgestate circulation for (a) the asymmetric reflection 1 , (b) the the asymmetric reflection 2, and (c) the full equilibration. The configuration with the local gate (d) fully covering and (e) partially covering the area between leads 6 and 8 .

We also measured $R_{D}\left(V_{L G}, V_{B G}\right)$ as plotted in Figure 3. Similar to the behavior of Figs. 2(a-d), Fig. 3(a) [Fig. 3(b)] resembles Fig. 3(d) [Fig. 3(c)] but with an inversion symmetry with Fig. 3(c) [Fig. 3(d)]. Each slice plot in Figs. 3(e-h) and the corresponding one in Figs. $2(\mathrm{e}-\mathrm{h})$ are qualitatively similar despite some quantitative differences. Moreover, in Figs. 3(e-h), one can see that $R_{D}$ is always the Hall resistance either for the region 1 $\left(R_{H 1}\right)$ or for the region $2\left(R_{H 2}\right)$. It is easily explained by the Kirchhoff's current-conservation relation, which points out that $R_{D}$ is the summation of $R_{H 1}$ or $R_{H 2}$ and the corresponding $R_{L}$. Consequently, symmetries imposed upon $R_{L}$ should appear in $R_{D}$.

Transport properties of the $\mathrm{QH}$ edge state can be conveniently analyzed by the Landauer-Büttiker formula 5.6.11 Based on the complete-mode-mixing hypothesis,, 11 one can distinguish three equilibration regimes: the asymmetric reflection $1\left(\nu_{1} \cdot \nu_{2}>0\right.$ and $\left.\left|\nu_{1}\right| \geq\left|\nu_{2}\right|\right)$, the asymmetric reflection $2\left(\nu_{1} \cdot \nu_{2}>0\right.$ and $\left.\left|\nu_{1}\right|<\left|\nu_{2}\right|\right)$, and the full equilibration $\left(\nu_{1} \cdot \nu_{2}<0\right)$ regimes. Figs. 4(a-c) illustrate the schematics of the edge state circulation in each regime for $\nu_{1}<0$ and $+H$. The asymmetric reflection 1 [Fig. 4(a)] is the case where the edge states inside the region 2 are not reflected at the interface. The situation is opposite for the asymmetric reflection 2 [Fig. 4(b)]. In full equilibration regime [Fig. 4(c)], the edge states in the regions 1 and 2 merge at the one side of the sample [for example, the upper side in Fig. 4(c)] and split at the other side [the lower side in Fig. 4(c)].

By evaluating scattering matrices, we calculated $R_{L}$ and $R_{D}$ as a function of $\nu_{1}$ and $\nu_{2}$ for different measurement conditions and equilibration regimes $\stackrel{5,6,11}{,}$ Detailed results are summarized below for $\nu_{1}<0$ and $+H$. (a) Asymmetric reflection $1\left(\nu_{1} \cdot \nu_{2}>0,\left|\nu_{1}\right| \geq\left|\nu_{2}\right|\right)$

$$
\begin{aligned}
& R_{D}=\left\{\begin{array}{ll}
\frac{h}{e^{2}} \frac{1}{\left|\nu_{2}\right|} & (\text { Configuration 1) } \\
-\frac{h}{e^{2}} \frac{1}{\left|\nu_{1}\right|} & (\text { Configuration 2) }
\end{array},\right. \\
& R_{L}= \begin{cases}\frac{h}{e^{2}} \frac{\left|\nu_{1}\right|-\left|\nu_{2}\right|}{\left|\nu_{1}\right|\left|\nu_{2}\right|} & \text { (Configuration 1) } \\
0 & \text { (Configuration 2) }\end{cases}
\end{aligned}
$$

(b) Asymmetric reflection $2\left(\nu_{1} \cdot \nu_{2}>0,\left|\nu_{1}\right|<\left|\nu_{2}\right|\right)$

$$
\begin{aligned}
& R_{D}=\left\{\begin{array}{ll}
\frac{h}{e^{2}} \frac{1}{\frac{\nu_{1} \mid}{2}} & (\text { Configuration 1) } \\
-\frac{h}{e^{2}} \frac{1}{\left|\nu_{2}\right|} & (\text { Configuration 2) }
\end{array},\right. \\
& R_{L}= \begin{cases}0 & \text { (Configuration 1) } \\
\frac{h}{e^{2}} \frac{\left|\nu_{2}\right|-\left|\nu_{1}\right|}{\left|\nu_{1}\right|\left|\nu_{2}\right|} & \text { (Configuration 2) }\end{cases}
\end{aligned}
$$

(c) Full equilibration $\left(\nu_{1} \cdot \nu_{2}<0\right)$

$$
\begin{aligned}
& R_{D}=\left\{\begin{array}{ll}
\frac{h}{e^{2}} \frac{1}{\left|\nu_{1}\right|} & (\text { Configuration 1) } \\
\frac{h}{e^{2}} \frac{1}{\left|\nu_{2}\right|} & (\text { Configuration 2) }
\end{array},\right. \\
& R_{L}= \begin{cases}0 & \text { (Configuration 1) } \\
\frac{h}{e^{2}} \frac{\left|\nu_{1}\right|+\left|\nu_{2}\right|}{\left|\nu_{1}\right|\left|\nu_{2}\right|} & \text { (Configuration 2) }\end{cases}
\end{aligned}
$$

In Eq. (1), $R_{L}$ for the configuration $2\left(=V_{38} / I\right)$ vanishes but it is finite in Eqs. (2) and (3), which is in coincidence with our experimental findings [compare Figs. 2(a) with $2(\mathrm{~b})$ for $\left.V_{B G}<\sim 55 \mathrm{~V}\right]$. Here, $V_{\alpha \beta}$ stands for the voltage difference between the leads $\alpha$ and $\beta$. The behavior of $R_{L}$ results because the EP in the asymmetric reflection 1 regime is different from that in the other regimes. In the former regime, the edge states are equilibrated at the lower boundary of the sample between the leads 5 and 6 [Fig. 4(a)], so that $V_{L}$ (or equivalently $R_{L}$ ) for the configuration $1\left(=V_{56}\right)$ is finite while the one for the configuration $2\left(=V_{38}\right)$ vanishes. On the contrary, in the asymmetric reflection 2 [Figs. 4(b)] and the full equilibration [Figs. 4(c)] cases, the equilibration takes place at the upper boundary of the sample between the leads 3 and 8 , resulting in the finite $R_{L}$ in the configuration 2 $\left(=V_{38} / I\right)$. Since the EP depends on the chiral direction of the edge-state circulation, $R_{L}$ in the configuration 1 (2) for $\nu_{1}<0$ becomes identical to the one in the configuration $2(1)$ for $\nu_{1}>0$ for an identical $H$. Equivalently, $R_{L}$ for $\nu_{1}<0$ in a negative $H$ becomes the same as that for $\nu_{1}>0$ in a positive $H$. It agrees with symmetries found in our experiment [Fig. 2]. Moreover, as shown as broken lines in Figs. 2(e-h), calculations and experiments are in a quantitative agreement with each other. It is important to point out that, in a p-n-p device, the EP's at opposite sides of the local-gate are located at different (upper or lower) boundaries of the sample. Thus, fourterminal $R_{L}$ of a graphene p-n-p device does not reveal the dependence on the location of EP, although the EP itself sensitively depends on the chiral direction of the $\mathrm{QH}$ edge state $\underline{\underline{11}}$ 
The calculated $R_{D}$ also coincides with the experimental value [Fig. 3], which is always $R_{H 1}$ or $R_{H 2}$. This can be explained by a simple Kirchhoff's law. For instance, $V_{36}$ is the summation of $V_{38}$ and $V_{86}\left(=R_{H 2} \times I\right)$ or equally $V_{35}\left(=R_{H 1} \times I\right)$ and $V_{56}$. As $V_{38}$ is zero in the asymmetric reflection 1 regime, $R_{D}$ for the configuration $1\left(=V_{36} / I\right)$ will be $R_{H 2}$ [Eq. (1)]. For the same reason, $V_{58} / I\left(=R_{D}\right.$ for the configuration 2) should be $-R_{H 1}$ [Eq. (1)]. In the other regimes, $V_{56}$ vanishes [Eqs. (2) and (3)], so that $R_{D}$ becomes $R_{H 1}$ for the configuration 1 and $-R_{H 2}$ for the configuration 2 .

Up to this point, we consider only the cases where all the regions between the leads 6 and 8 are covered by local gates [Fig. 4(d)]. However, as seen in Fig. 4(e), the regions near the leads 6 and 8 may not be fully covered by the local gates, which results in the formation of unintended p-n interfaces around the regions [dotted squares in Fig. 4(e)]. This may change the conductance features we discuss above. Apparently, the conductance change takes place in a two-terminal configuration as the additional interface forms a p-n-p junction between the source and the drain. But, in the following discussion, we demonstrate that the results of four-terminal measurements are not affected. This implies that the difference in the chemical potential $(\mu)$ between the point A and the lead $6\left(\mu_{A}-\mu_{6}\right)$ remains the same for both fully and partially covered cases, when the leads 6 and 8 are a voltage probe and a drain, respectively [here, we focus on the configuration 1 in Fig. 1(c) but the same argument is valid for the configuration 2]. As the chemical potential does not change across the voltage probe without current flow, it is straightforward to show that $\mu_{6}$ is always identical to $\mu_{B}$ and $\mu_{C}$ for both cases. In addition, the Hall voltage inside the region $2, \mu_{A}-\mu_{B}$, does not vary irrespective of the presence or the absence of p-n interfaces outside the region 2 (the region covered by the local gate). Consequently, the presence of additional bipolar interfaces does not affect the value $\mu_{A}-\mu_{6}$, neither the corresponding four-terminal resistances. One notes that, for the partially covered case, $\mu_{B}$ can differ from $\mu_{8}$ in certain equilibration regimes so that the partial coverage of the top gate affects the two-terminal results. This demonstrates the high benefit of the multi-terminal measurements in comparison with the two-terminal ones for edge-state equilibration studies.

In this study, we independently controlled the local filling factors of the top-gated and the rest of back-gated region of a graphene sheet. It enabled us to access to diverse combination of bipolar configurations in the sheet along with more systematic examination of equilibration condition of QH edge conducting states. We have shown that both the longitudinal ( $R_{L}$ in Fig. 2) and the diagonal $\left(R_{D}\right.$ in Fig. 3) resistances, obtained in four-terminal configurations, of this locally top-gated hybrid p-n junction device are asymmetric with respect to the zero point. The behaviors of $R_{L}$ and $R_{D}$ are also in clear contrast to the two-terminal studies on locally top-gated $\mathrm{p}-\mathrm{n}^{\underline{8}}$ and $\mathrm{p}-\mathrm{n}-\mathrm{p} \frac{10}{\underline{\underline{n}}}$ devices of graphene as well as to similar fourterminal studies on locally top-gated $\mathrm{p}$-n-p devices $\stackrel{11}{=}$ The resistances in two different measurement configurations [Figs. 1(c,d)] and $H$ directions confirms that the asymmetry is caused by the dependence of the four-terminal resistance of a graphene $\mathrm{p}-\mathrm{n}$ interface on the location of the EP, which varies with the chiral direction of the $\mathrm{QH}$ edge state. Since two-terminal conductances do not depend on the chiral direction and the EP's in a p-n$\mathrm{p}$ device are always located at opposite boundaries of the sample, the location of the EP could not be traced in the previous experiments and the resistances turned

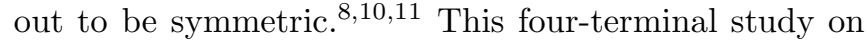
a graphene p-n interface provides a deeper insight into how and where the $\mathrm{QH}$ edge-states are equilibrated in a locally gated graphene system, which is essential in designing devices such as quantum interferometers out of graphene.

\section{Acknowledgments}

This work was supported by National Research Foundation of Korea through Acceleration Research Grant (No. R17-2008-007-01001-0) and by the Ministry of Education, Science and Technology under the grant No. 2009-0083380. BO acknowledges support from Singapore National Research foundation under NRF Award No. NRF-RF2008-07 and by NUS NanoCore.
* Corresponding author: hjlee@postech.ac.kr

${ }^{1}$ K. S. Novoselov, A. K. Geim, S. V. Morozov, D. Jiang, M. I. Katsnelson, I. V. Grigorieva, S. V. Dubonos, and A. A. Firsov, Nature (London) 438, 197 (2005).

${ }^{2}$ Y. Zhang, Y. W. Tan, H. L. Stormer, and P. Kim, Nature (London) 438, 201 (2005).

3 D. K. Ki, S. Jo, and H. J. Lee, Appl. Phys. Lett. 94, 1621113 (2009).

4 B. I. Halperin, Phys. Rev. B 25, 2185 (1982).

5 M. Büttiker, Phys. Rev. B 38, 9375 (1988).

6 See a review in C. W. J. Beenakker and H. van Houten,
Solid State Phys. 44, 1 (1991); also found in e-print arXiv:0412664v1.

7 M. I. Katsnelson, K. S. Novoselov, and A. K. Geim, Nature Phys. 2, 620 (2006).

8 J. R. Williams, L. DiCarlo, and C. M. Marcus, Science 317, 638 (2007).

9 D. A. Abanin and L. S. Levitov, Science 317, 641 (2007).

10 B. Özyilmaz, P. Jarillo-Herrero, D. Efetov, D. A. Abanin, L. S. Levitov, and P. Kim, Phys. Rev. Lett. 99, 166804 (2007).

11 D. K. Ki and H. J. Lee, Phys. Rev. B 79, 195327 (2009). 
12 T. Lohmann, K. v. Klitzing, and J. H. Smet, Nano. Lett. 9, 1973 (2009).

13 K. S. Novoselov, A. K. Geim, S. V. Morozov, D. Jiang, Y. Zhang, S. V. Dubonos, I. V. Grigorieva, and A. A. Firsov, Science 306, 666 (2004).
14 B. Huard, J. A. Sulpizio, N. Stander, K. Todd, B. Yang, and D. Goldhaber-Gordon, Phys. Rev. Lett. 98, 236803 (2007). 